\title{
Accurate Binding of Sodium and Calcium to a POPC Bilayer by Effective Inclusion of Electronic Polarization
}

\section{Melcr, Josef}

2018-04-26

Melcr , J , Martinez-Seara , H , Nencini , R , Kolafa , J , Jungwirth , P \& Ollila , O H S 2018 , ' Accurate Binding of Sodium and Calcium to a POPC Bilayer by Effective Inclusion of Electronic Polarization ' , Journal of Physical Chemistry B , vol. 122 , no. 16 , pp. 4546-4557 . https://doi.org/10.1021/acs.jpcb.7b12510

http://hdl.handle.net/10138/324024

https://doi.org/10.1021/acs.jpcb.7b12510

acceptedVersion

Downloaded from Helda, University of Helsinki institutional repository.

This is an electronic reprint of the original article.

This reprint may differ from the original in pagination and typographic detail.

Please cite the original version. 


\section{Accurate Binding of Sodium and Calcium to a POPC Bilayer by Effective Inclusion of Electronic Polarization}

Josef Melcr,${ }^{\dagger}$ Hector Martinez-Seara,${ }^{\dagger}$ Ricky Nencini, ${ }^{\dagger}$ Jiř́ Kolafa,,${ }^{\ddagger}$ Pavel Jungwirth, ${ }^{\dagger, \uparrow}$ and O. H. Samuli Ollila ${ }^{*, \dagger, \S}$

$\dagger$ Institute of Organic Chemistry and Biochemistry, Academy of Sciences of the Czech Republic, Prague 6, Czech Republic

$\ddagger$ Department of Physical Chemistry, Institute of Chemical Technology, Prague 6, Czech Republic

IDepartment of Physics, Tampere University of Technology, P.O. Box 692, FI-33101 Tampere,

Finland

§Institute of Biotechnology, University of Helsinki

E-mail: samuli.ollila@helsinki.fi 


\begin{abstract}
Binding affinities and stoichiometries of $\mathrm{Na}^{+}$and $\mathrm{Ca}^{2+}$ ions to phospholipid bilayers are of paramount significance in the properties and functionality of cellular membranes. Current estimates of binding affinities and stoichiometries of cations are, however, inconsistent due to limitations in the available experimental and computational methods. In this work, we improve the description of the binding details of $\mathrm{Na}^{+}$and $\mathrm{Ca}^{2+}$ ions to the 1-Palmitoyl-2oleoyl-phosphatidylcholine (POPC) bilayer by implicitly including electronic polarization as a mean field correction, known as the electronic continuum correction (ECC). This is applied by scaling the partial charges of a selected state-of-the-art POPC lipid model for molecular dynamics simulations. Our improved ECC-POPC model reproduces not only the experimentally measured structural parameters for the ion-free membrane, but also the response of lipid head group to a strongly bound cationic amphiphile, as well as the binding affinities of $\mathrm{Na}^{+}$ and $\mathrm{Ca}^{2+}$ ions. With our new model we observe on the one side negligible binding of $\mathrm{Na}^{+}$ ions to POPC bilayer, while on the other side stronger interactions of $\mathrm{Ca}^{2+}$ primarily with phosphate oxygens, which is in agreement with the previous interpretations of the experimental spectroscopic data. The present model results in $\mathrm{Ca}^{2+}$ ions forming complexes with one to three POPC molecules with almost equal probabilities, suggesting more complex binding stoichiometries than those from simple models used to interpret the NMR data previously. The results of this work pave the way to quantitative molecular simulations with realistic electrostatic interactions of complex biochemical systems at cellular membranes.
\end{abstract}

\title{
Introduction
}

Interactions of ions with cellular membranes play a key role in many important biological processes. ${ }^{1,2}$ Ions, especially multivalent cations, modify general properties of the membrane which also modulate the embedded transmembrane proteins. ${ }^{2-4}$ For example, $\mathrm{Ca}^{2+}$ is crucial in propagation of neural signals. It promotes membrane fusion by bridging lipids in the vesicles carrying neurotransmitters and the neuron synapsis membrane. ${ }^{5,6} \mathrm{Ca}^{2+}$ also participates in the T-cell receptor activation. It induces the detachment of the positively charged cytosolic tails of the CD3 
protein complex from the negatively charged intracellular membrane making it accessible for the Lck protein. ${ }^{7}$ Another example involving sugar-lipid interactions mediated by ions concerns $\mathrm{Ca}^{2+}$ modulating the presentation of the sugars present in the $\mathrm{PI}(4,5) \mathrm{P}_{2}$ lipid which ultimately modulates the phospholipase $\mathrm{C}$ delta 1 pleckstrin homology domain (PLC $\delta 1-\mathrm{H}){ }^{8}$ Interestingly, this effect is not present for $\mathrm{Mg}^{2+}$, which illustrates the selectivity of these processes with respect to particular ions. Despite our increasing understanding of the role of ions in cell membrane-related processes the molecular details of the mechanisms behind such processes remain elusive in many cases.

Direct measurements of ion-membrane interactions in complex biological systems are difficult. Hence, simplified lipid bilayers are often used as entry-level models to shed light on the role of ions in complex biological membranes. ${ }^{1,2,9}$ For this reason, interactions of biologically relevant cations, in particular $\mathrm{Na}^{+}$and $\mathrm{Ca}^{2+}$, with zwitterionic phosphocholine (PC) bilayers have been widely studied experimentally ${ }^{1-4,10-13}$ and using molecular dynamics (MD) simulations. ${ }^{14-25}$ The details of ion binding are, however, not fully consistent in the literature. Non-invasive spectroscopic methods, like nuclear magnetic resonance (NMR), scattering, and infrared spectroscopies mostly suggest that $\mathrm{Na}^{+}$ions exhibit negligible binding to PC lipid bilayers. In contrast, $\mathrm{Ca}^{2+}$ is observed to specifically bind to a couple of PC molecules via their phosphate groups. ${ }^{4,10-13,26-28}$ Most atomistic resolution MD simulation models predict stronger membrane bindings of the cations than observed in experiments. ${ }^{29}$ Namely, simulations report various degrees of $\mathrm{Na}^{+}$accumulation at the lipid interface ${ }^{14}$ and for $\mathrm{Ca}^{2+}$ strong binding to up to four PC lipids simultaneously, involving not only interactions with phosphates but also with carbonyl oxygens. ${ }^{15,24,25}$

Recent studies within the NMRlipids project (nmrlipids.blogspot.fi) ${ }^{29}$ made an attempt to resolve these disagreements. A direct comparison of ion binding affinities to PC bilayers between simulations and experiments has been made possible using the electrometer concept. ${ }^{30}$ Namely, the changes in NMR order parameters of the head group upon addition of ions are directly compared to the MD simulations results. Analyzing massive amounts of data collected within an open collaboration approach, it was concluded that the accuracy of the current state-of-the-art lipid models for MD simulations is not sufficient for a detailed interpretation of the interactions of 
cations with PC lipid bilayers. ${ }^{29}$

In this work, we improve the description of cation binding to a POPC bilayer within MD simulations via an implicit inclusion of electronic polarizability in the polar region of phospholipids. To this end, we apply the electronic continuum correction (ECC), ${ }^{31}$ within which scaling of the atomic partial charges is employed to account for the missing electronic polarizability in standard force field MD simulations. Such an approach has been shown previously to improve the behavior of ions in simulations of aqueous salt solutions. ${ }^{32-35}$ Here, we extend the ECC approach to aqueous lipid membranes taking the Lipid14 force field ${ }^{36}$ as a starting point for refining the POPC model. This choice is justified by the fact that the Lipid14 provides the least inaccurate descriptions of cation binding among the existing force fields. ${ }^{29}$ The newly developed ECC-POPC model reproduces the experimentally measurable structural parameters of an ion-free POPC lipid bilayer with the accuracy comparable to the best state-of-the-art lipid models, while at the same time significantly improving the membrane binding affinities of sodium and calcium cations.

\section{Methods}

\section{Electronic continuum correction for lipid bilayers}

The lack of electronic polarizability in standard MD force fields has been considered a serious issue since the early days of lipid bilayer simulations. In this work, we circumvent the demanding explicit inclusion of electronic polarization effects ${ }^{37,38}$ by accounting for the electronic part of polarizability in lipid bilayer simulations implicitly via the electronic continuum correction (ECC). ${ }^{31}$ Technically, this is similar to the phenomenological charge-scaling applied in earlier studies of surfactants, lipids or ionic liquids. ${ }^{39-41}$ However, the present concept of ECC is physically well justified and has theoretical support. ${ }^{31,42-44}$

According to ECC, the electronic polarizability can be included into non-polarizable MD in a mean-field way by embedding the ions in a homogeneous dielectric continuum with a dielectric constant $\epsilon_{e l}$, which is the electronic part of the dielectric constant of the medium. ${ }^{31}$ Following 
Coulomb's law, ECC can be directly incorporated by scaling the charges with a constant scaling factor of $f_{q}=\epsilon_{e l}^{-1 / 2}$, yielding

$$
Q^{E C C}=f_{q} \cdot Q
$$

for the ECC corrected charges. Given that the high frequency dielectric constant of water is $\epsilon_{e l}=1.78$ (i.e., the square of the refraction index), the scaling factor for ions in water is roughly $f_{q} \approx 0.75$. This scaling factor significantly improves the accuracy of simulations of solvated ions, when quantitatively compared with neutron scattering data. ${ }^{32-35}$ It is important to note that the value of the high frequency dielectric constant is around 2 for almost any biologically relevant environment. ${ }^{31}$ The dielectric discontinuity in a lipid bilayer thus arises only from the orientational polarization of the molecules, which is accounted for explicitly in standard MD simulations. Therefore, the same correction for the electronic polarizability can be applied throughout the lipid bilayer/aqueous solution interface.

While using the scaling factor of $f_{q}=0.75$ for ions in water is well justified in the ECC theory, ${ }^{31}$ it is not clear whether the same factor should be applied to partial charges used to describe molecules in MD models, e.g., lipids in our case. ${ }^{44}$ Unlike the total charge of an ion, atomic partial charges within molecules are not physical observables. Several computational schemes exist for the assignment of partial charges for biomolecules ${ }^{45}$ with the restrained electrostatic potential method (RESP) being commonly used ${ }^{46,47}$ Considering that water is often included in RESP calculations or charges are refined to improve certain experimental observables, the electronic polarizability effects of the solvent may to some extent be included in standard force fields. ${ }^{46-50}$ Thus, our application of the scaling factor, $f_{q}$, to existing partial charges in molecules does not necessarily follow $f_{q}=\epsilon_{e l}^{-1 / 2}$. Instead, a consistent scaling factor should lie between the value of 0.75 (i.e., no electronic polarizability included in the original partial charges) and 1 (i.e., electronic polarizability fully included in the original partial charges).

Here we develop a ECC-POPC lipid model that accurately describes binding of sodium and calcium ions to the POPC lipid bilayer. The Lipid $14^{36}$ force field (available in a Gromacs format from Ref. 51) was used as a starting point since it provides the most accurate response of the 
head groups to ions among the available lipid models (see Figs. 2 and 5 in Ref. 29). Additionally, the Lipid14 model provides relatively realistic head group, glycerol backbone, and acyl chain structures. ${ }^{36,52}$ We applied the ECC correction to the Lipid14 model of POPC by scaling partial charges of the head group, glycerol backbone, and carbonyl regions. These are the polar parts of phospholipids which can contribute to the cation binding. A similar approach is also applied to the CHARMM36 model of POPC ${ }^{53}$ in the the SI.

To reproduce the experimental ion binding affinities, we scanned possible values of the scaling factor from the interval $f_{q} \in(0.75,1.0)$. The ion binding affinity was benchmarked against experiments using the head group order parameters and the electrometer concept, ${ }^{29,30}$ as discussed more detail in the next section. Scaling down the partial charges reduced the ion binding affinity. We found the most accurate ion binding affinities with a scaling factor of $f_{q}=0.8$, which is only slightly higher than the ECC one $\left(f_{q}=0.75\right)$. Note that common empirical scaling factors for monovalent ions in water are 0.8 or even closer to unity. ${ }^{50,54,55}$ In contrast, modern force fields for ionic liquids suggest values of $0.6-0.65$, which are lower than $\epsilon_{e l}^{-1 / 2} .{ }^{56}$ Directly applying the 0.8 scaling to the partial charges of the head group, the glycerol backbone, and the carbonyls reduced the area per lipid to $60 \AA^{2}$. This area is smaller than in the original Lipid14 model (65.6土 $\left.0.5 \AA^{2}\right)^{36}$ and in experiments $\left(64.3 \AA^{2}\right) .{ }^{57}$ The decrease of the area per lipid arises from a reduced hydration of the lipid head group region after scaling of the partial charges, which effectively reduces the head group polarity. We solve this problem by slightly reducing the effective radii of the modified head group atoms via lowering the $\sigma$ parameters in the Lennard-Jones potential by a factor of $f_{\sigma}=0.89$. The same approach was successfully adopted for the ECC ions in aqueous

solutions previously. ${ }^{32-35}$ After reducing the head group atoms $\sigma$ parameters, the area per molecule is restored to the experimental value (Table 1).

\section{Electrometer concept}

Comparing MD simulation to NMR experiments, we can validate the ion binding affinity in lipid bilayer simulations using the 'electrometer concept' . 29,30 This method is based on the experimental 
observation that the $\mathrm{C}-\mathrm{H}$ bond order parameters of $\alpha$ and $\beta$ carbons in a PC lipid head group (Fig. 1) are proportional to the amount of charge bound per lipid. ${ }^{30}$ The order parameters for all $\mathrm{C}-\mathrm{H}$ bonds in lipid molecules can be accurately measured using ${ }^{2} \mathrm{H}$ NMR or ${ }^{13} \mathrm{C}$ NMR techniques. ${ }^{58}$ From MD simulations the order parameters can be calculated using the relation

$$
S_{\mathrm{CH}}=\frac{1}{2}\left\langle 3 \cos ^{2} \theta-1\right\rangle,
$$

where $\theta$ is the angle between the $\mathrm{C}-\mathrm{H}$ bond and the membrane normal. Angular brackets point to the average over all sampled configurations.

The relation between the amount of the bound charge per lipid, $X^{ \pm}$, and the head group order parameter change, $\Delta S_{\mathrm{CH}}^{i}$, is empirically quantified as ${ }^{30,59}$

$$
\Delta S_{\mathrm{CH}}^{i}=S_{\mathrm{CH}}^{i}\left(X^{ \pm}\right)-S_{\mathrm{CH}}^{i}(0) \approx m_{i} \frac{4}{3 \chi} X^{ \pm}
$$

where $i$ refers to either the $\alpha$ or $\beta$ carbons, $S_{\mathrm{CH}}^{i}(0)$ denotes the order parameter in the absence of bound charge, $\chi$ is the quadrupole coupling constant $(\chi \approx 167 \mathrm{kHz})$, and $m_{i}$ is an empirical constant depending on the valency and location of the bound charge.

The measured change of the order parameter depends on the head group response to the bound charge and on the amount of the bound charge (i.e., $m_{i}$ and $X^{ \pm}$in Eq. 3, respectively). The empirical factor $m_{i}$ has to be adequately quantified before the electrometer concept can be used to analyze the binding affinities. This calibration has been done experimentally for a wide range of systems. ${ }^{30,60}$ To calibrate the response of the head group order parameters to the bound charge in simulations, we use experimental data for a strong cationic surfactant dihexadecyldimethylammonium bromide (DHAB) mixed with a POPC bilayer. ${ }^{61} \mathrm{DHAB}$

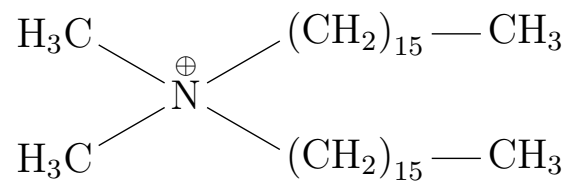

is a cationic surfactant having two acyl chains and bearing a unit charge at the hydrophilic end. 
Due to its structure it is expected to locate in the bilayer similarly to the phospholipids and its molar ratio then gives directly the amount of bound unit charge per lipid $X^{ \pm}$in these systems. ${ }^{61}$

\section{Salt concentrations and binding affinities}

When measuring head group order parameters, the NMR experiments report the employed salt concentrations in two different ways. Some studies provide the salt concentrations in water before solvating the lipids, ${ }^{10} C_{i o n}^{\prime}$, while others use atomic absorption spectroscopy and report the salt concentration in the supernatant after the solvation of lipids, ${ }^{11} C_{i o n}$. In this work, we stick to the latter definition by estimating the salt concentration in the aqueous bulk region using the farthest point from the lipid bilayer in the aqueous phase. Note that the former definition was used by some of us previously. ${ }^{29}$ Although these two definitions provide somewhat different values when applied to $\mathrm{Ca}^{2+}$ concentrations, their particular choice does not affect the conclusions of this work in any significant way.

To quantify the ion binding affinities to a lipid bilayer, we calculate the relative surface excess of ions with respect to water, $\Gamma_{i o n}^{\text {water }}{ }^{62}$ Such a quantity compares the adsorption of ions to the adsorption of water molecules at the interface without the necessity of defining a Gibbs dividing surface between the membrane interior and the water bulk region. In our simulations, we only assume that the interface is located between the ion-free hydrophobic interior of the lipid bilayer and the aqueous region far from the membrane. Such a setup and the above definition of bulk ion concentrations provides a simple relation for the relative surface excess $\Gamma_{i o n}^{\text {water }}$ for simulations of lipid bilayers,

$$
\Gamma_{\text {ion }}^{\text {water }}=\frac{1}{2 A_{b}}\left(n_{\text {ion }}-n_{\text {water }} \frac{C_{\text {ion }}}{C_{\text {water }}}\right) .
$$

Here, $n_{\text {water }}$ and $n_{\text {ion }}$ are the total numbers of water molecules and ions in the system, $C_{\text {water }}$ and $C_{i o n}$ are their respective bulk concentrations in the aqueous phase (estimated from the solution density of farthest point between periodic bilayers), and $A_{b}$ is the area of the unit cell in the membrane plane. The total area of the interface is then twice the area of the membrane, i.e., $2 A_{b}$, because the 
bilayer has an interface at each of the two leaflets.

\section{Validation of lipid bilayer structure against NMR and scattering experiments}

The structures of lipid bilayers in simulations without ions were validated against NMR by calculating the order parameters for the $\mathrm{C}-\mathrm{H}$ bonds and against $\mathrm{X}$-ray scattering experiments by evaluating the scattering form factors. NMR order parameters validate the structures sampled by the individual lipid molecules with atomic resolution. The simulated order parameters were calculated for all $\mathrm{C}-\mathrm{H}$ bonds in lipid molecules from Eq. 2. Scattering form factors validate the dimensions of the lipid bilayer (i.e., the bilayer thickness and area per molecule). Form factors were calculated using a relation

$$
F(q)=\int_{-D / 2}^{D / 2}\left(\rho_{e l}(z)-\rho_{e l}^{s}\right) \cos \left(z q_{z}\right) \mathrm{d} z
$$

where $\rho_{e l}(z)$ is the total electron density, $\rho_{e l}^{s}$ is the electron density of the solvent far in the aqueous bulk, and $z$ is the distance from the membrane center along its normal with $D / 2$ being half of the unit cell size.

\section{Simulation details}

\section{Simulations of POPC bilayers with aqueous ions}

Simulations of a POPC bilayer in pure water or at varying salt concentrations consisted of 128 POPC molecules and approximately 50 water molecules per lipid in an orthorhombic simulation box with periodic boundary conditions. The $\mathrm{SPC} / \mathrm{E}^{63}$ water model was used in all ECC-POPC model simulations reported in the main text. This model was also used in the previous parametrization of ECC-ions ${ }^{32,33,35}$ because its lowered dielectric constant is consistent with the ECC concept. ${ }^{31,44}$ The robustness of our approach with other water models (OPC, ${ }^{64}$ OPC $3,{ }^{65}$ TIP3P, ${ }^{66}$ TIP3P-FB and TIP4P-FB, ${ }^{67}$ and TIP4P/2005 ${ }^{68}$ ) was verified and is presented in the Supporting Information (SI). Sodium, calcium, and chloride ions were modeled as ECC-ions with parameters 
from Refs. 32,33. Scaled charges and Lennard-Jones radii for atoms forming the POPC lipid were derived in this work starting from the Lipid14 force field. ${ }^{36}$ For comparison, simulations with the Lipid14 model $^{36}$ and ion models by Dang and coworkers ${ }^{69-71}$ or ECC-ions ${ }^{32,33,35}$ were also performed. The TIP3P water model ${ }^{66}$ was used in all simulations with the original Lipid14 model. Simulation data for the Lipid14 model with Åqvist ions ${ }^{72}$ and the TIP3P ${ }^{66}$ water model were taken directly from Refs. 29,73-76.

MD simulations were performed using the GROMACS ${ }^{77}$ simulation package (version 5.1.4). The simulation parameters used in this work are summarized in Table S1 in the SI. Compatibility with the openMM simulation package ${ }^{78}$ was also tested and is presented in the SI. For deposited trajectories and parameter files see Refs. 79-81.

\section{Simulations of POPC bilayers with cationic surfactants}

The topology of the dihexadecyldimethylammonium cation was created with the automated topology builder. ${ }^{82}$ The AmberTools program ${ }^{83}$ was used to generate the Amber-type force field parameters. These parameters were then converted to the Gromacs format with the acpype tool. ${ }^{84}$ The partial charges were manually modified in the non-scaled version to approximately match similar segments in Lipid14. ${ }^{36}$ For simulations of cationic surfactants mixed with ECC-POPC, all charges of cationic surfactants were scaled by a factor $f_{q}=0.75$ consistently with the counterions. The Lennard-Jones radii were scaled with the same factor as for ECC-POPC, i.e. $f_{\sigma}=0.89$.

The cationic surfactants were randomly mixed among the phospholipids to form bilayer structures with mole fractions of $10 \%, 20 \%, 30 \%, 42 \%$, or $50 \%$ of surfactant in the POPC bilayer. All these systems contained 50 POPC molecules per leaflet, 6340 water molecules, and 6, 14, 21, 35, or 50 surfactants per leaflet. Chloride counter ions were used in all the simulations, while bromide was the counter ion in the experiment. ${ }^{61}$ For POPC, either Lipid14 with TIP3P ${ }^{66}$ or ECC-POPC with $\mathrm{SPC} / \mathrm{E}^{63}$ parameters were used. The first $20 \mathrm{~ns}$ of the total simulation time of $200 \mathrm{~ns}$ was considered as an equilibration period and was thus omitted from the analysis. We checked that a sufficient lipid exchange occurred during the simulations. For deposited trajectories and parameter 

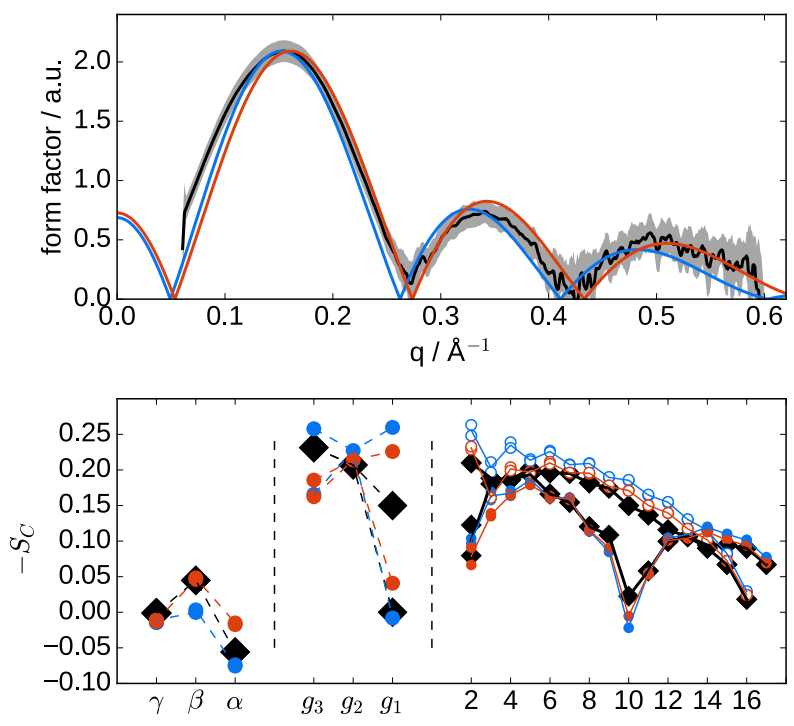

Figure 1: Top: X-ray scattering form factors from simulations with the Lipid $14^{36}$ and the ECCPOPC models compared with experiments ${ }^{57}$ at $303 \mathrm{~K}$.

Middle: Order parameters of POPC head group, glycerol backbone and acyl chains from simulations with the Lipid $14^{36}$ and the ECC-POPC models compared with experiments ${ }^{92}$ at $300 \mathrm{~K}$. The size of the markers for the head group order parameters correspond to the error estimate \pm 0.02 for experiments, ${ }^{52,58}$ while the error estimate for simulations is \pm 0.005 (Bayesian estimate of $95 \%$ confidence interval $^{93}$ ). The size of the points for acyl chains are decreased by a factor of 3 to improve the clarity of the plot. Open/closed symbols are used for palmitoyl/oleoyl chains of POPC. Bottom: The chemical structure of POPC and the labeling of the carbon segments.

files of scaled and unscaled simulations see Refs. 85 and 86-91, respectively.

\section{Results and Discussion}

\section{Structural parameters of a pure ECC-POPC model membrane: Agreement with experiments}

First, we present results for bilayers in pure water. The ECC-POPC and Lipid14 models both reproduce the experimental X-ray scattering form factors of a POPC bilayer with a comparable 
Table 1: Values of the area per lipid (APL) of POPC bilayers without ions.

\begin{tabular}{l|cc} 
model & APL $\left(\AA^{2}\right)$ & Temperature $[\mathrm{K}]$ \\
\hline Lipid14 & $65.1 \pm 0.6$ & 300 \\
Lipid14 & $65.6 \pm 0.5$ & 303 \\
\hline ECC-POPC & $63.2 \pm 0.6$ & 300 \\
\hline experiment $^{57}$ & 64.3 & 303 \\
\hline
\end{tabular}

accuracy (see Fig. 1). The area per lipid from the Lipid14 model is by $\approx 1 \AA$ larger than the experimental value in Table 1 , while the value from the ECC-POPC model is by $\approx 1 \AA$ smaller than the experimental one. The values of the area per lipid of the ECC-POPC model vary slightly when simulated with different water models (i.e., within the interval of 62.2-66.8 A, see Table S2 in SI), while still being close to the experimentally reported values. We can thus conclude that the ECCPOPC model reproduces the experimental dimensions of the POPC lipid bilayer with a comparable accuracy to other state-of-the-art lipid models. ${ }^{58}$

Similarly, the acyl chain order parameters of the ECC-POPC model, as well as those of the Lipid14 model, ${ }^{36}$ agree with the experimental values within the error bars, as presented in Fig. 1. Notably, the experimentally measured forking and small order parameter values of the $C_{2}$ segment in $s n-2$ chain are well reproduced by both models. This feature has been suggested to indicate that the carbonyl of the $s n-2$ chain is directed towards the water phase, in contrast to the carbonyl in the $s n-1$ chain, which orients more along the bilayer plane. ${ }^{94-96}$ This arrangement, which is not fully reproduced by other available lipid models, ${ }^{58}$ may be a relevant feature for the ion binding details.

The order parameters of the $\alpha$ and $\beta$ carbons in the head group are slightly larger in the ECCPOPC model than in the Lipid14 model, which is apparently related to the P-N vector orienting by about $7^{\circ}$ more toward the water phase in the former model, see Fig. 2 . While both models perform relatively well, considering the available experimental evidence, it is not possible to decide which of the two models provides more realistic head group orientations. The ECC-POPC model gives the $\beta$ carbon order parameter value closer to experiments than the Lipid14 model, while the opposite is true for the $\alpha$ carbon. The accuracy of both models in the glycerol backbone region is comparable to other state-of-the-art lipid model available in literature, ${ }^{52}$ see Fig. 1 . The 
Figure 2: The changes of head group order parameters and $\mathrm{P}-\mathrm{N}$ vector orientation as a function of a molar fraction of the cationic surfactant dihexadecyldimethylammonium in a POPC bilayer from simulations and experiments ${ }^{61}$ at $313 \mathrm{~K}$.

electrostatic potentials as obtained in ECC-POPC and Lipid14 simulations are compared in the SI.

\section{Calibration of head group response to membrane-bound charge using cationic surfactant}

Before studying the sodium and calcium ion binding affinities, we quantify the response of the head group order parameters to the amount of bound charge by using mixtures of monovalent cationic surfactants (dihexadecyldimethylammonium) and POPC. ${ }^{61}$ These mixtures have a welldefined amount of bound charge per PC, namely, the molar fraction of cationic surfactants. This is due to the ability of dihexadecyldimethylammonium to directly insert in the lipid bilayer due to its two hydrophobic acyl chains. Furthermore, available experimental data for these systems can be 
used to validate the sensitivity of lipid head group order parameters to the amount of bound charge in simulations. ${ }^{61}$

The changes of the head group order parameters with increasing amount of the cationic surfactant are compared between simulations and experiments ${ }^{61}$ in Fig. 2. An approximately linear decrease of the order parameters, as expected from Eq. 3, is observed in both simulations and experiments at least for mole fractions below $\sim 30 \%$. The slope is, however, too steep in the Lipid14 model indicating that the response of head group order parameters to the bound positive charge is too strong. In contrast, the slope of the ECC-POPC model is in a very good agreement with experiments for the $\alpha$ segment, while being only slightly underestimated for the $\beta$ segment.

In Fig. 2, we show the headgroup P-N vector angle as a function of the mole fraction of the cationic surfactant. As suggested previously ${ }^{30}$ and observed in MD simulations, ${ }^{18,19,97,98}$ the headgroup orients more towards the water phase with the increasing amount of positive charge in the PC lipid bilayer. The effect is more pronounced in the Lipid14 model than in the ECC-POPC model. For example, the addition of $50 \%$ mole fraction of the cationic surfactant leads to a decrease of $20^{\circ}$ of the P-N vector angle for the Lipid14 model while only of $11^{\circ}$ in the ECC-POPC model. The difference is in line with the smaller order parameter changes and the reduced charge-dipole interactions in the latter model. The weaker sensitivity of the P-N vector angle response in the ECC-POPC model is in better agreement with experiments.

\section{Validation of ECC-POPC model using binding affinities to $\mathrm{Na}$ and $\mathrm{Ca}$ cations: the electrometer concept}

Changes of the lipid bilayer head group order parameters extracted from simulations and experiments ${ }^{10,11}$ are shown in Figs. 3 and 4 as functions of $\mathrm{NaCl}$ or $\mathrm{CaCl}_{2}$ concentrations. As seen in Fig. 2, the order parameters decrease proportionally to the amount of the bound positive charge. These results can be thus used to compare the ion binding affinities to lipid bilayers between simulations and experiments using the electrometer concept. ${ }^{29,30}$

The experimentally measured small order parameter changes with $\mathrm{NaCl}$ (Fig. 3) are reproduced 
Figure 3: Changes of the head group order parameters of a POPC bilayer as a function of $\mathrm{NaCl}$ concentration in bulk $\left(C_{i o n}\right)$ from simulations with different force fields at $313 \mathrm{~K}$ together with experimental data for DPPC $(323 \mathrm{~K}){ }^{10}$ and POPC $(313 \mathrm{~K}) .{ }^{11}$ Simulation data with Lipid14 and Åqvist ion parameters at $298 \mathrm{~K}$ are taken directly from Refs. ${ }^{73,76}$

by the Lipid14 model simulated with Åqvist ions. However, the same combination of models overestimates the order parameter changes with $\mathrm{CaCl}_{2}$ (Fig. 4). Replacing Åqvist ions with ion parameters by Dang et al. ${ }^{69-71}$ or ECC-ions ${ }^{32,33,35}$ did not improve the results (Figs. 3 and 4). In line with the previous work, ${ }^{29}$ the results suggest that improvements in the lipid parameters are required to correctly describe the binding of cations to phospholipid bilayers.

The results from simulations combining the ECC-POPC with the ECC-ion models ${ }^{32,33,35}$ exhibit a significantly improved behavior of the POPC head group order parameters as a function of $\mathrm{NaCl}$ or $\mathrm{CaCl}_{2}$ concentrations, see Fig. 3 and Fig. 4. Considering that we are also able to reproduce the experimental response in systems with known charge density (see above section), we conclude that our ECC model correctly reproduces the binding affinities of $\mathrm{Na}^{+}$and $\mathrm{Ca}^{2+}$ ions to the POPC lipid bilayer. Also the binding affinity of $\mathrm{K}^{+}$ions to POPC bilayer is in line with the experimental data $^{12,99,100}$ (shown in SI). Furthermore, while the response of the glycerol backbone $g_{3}$ order 
Figure 4: Changes of the head group order parameters and P-N vector orientation of a POPC bilayer as a function of the $\mathrm{CaCl}_{2}$ concentration in bulk $\left(C_{i o n}\right)$ from simulations at $313 \mathrm{~K}$ together with experimental data (DPPC $(323 \mathrm{~K})^{10}$ and POPC $\left.(313 \mathrm{~K})^{11}\right)$. The error estimate for bulk concentrations is approximately $10 \mathrm{mM}$. The order of magnitude larger error in the simulation with Lipid14 and ECC-ions is due to unconverged bulk densities (shown if Fig. 5) limited by the simulation box. Simulation data with Lipid14 and Åqvist ion parameters at $298 \mathrm{~K}$ are taken directly from Refs. 73-75.

parameter to $\mathrm{CaCl}_{2}$ was significantly overestimated in the original Lipid14 model, the ECC-POPC model provides an improved agreement with experiment, as seen in Fig. 4. Also the changes of the 
Table 2: Concentration of $\mathbf{C a}^{2+}$ with respect to water $\left(C_{i o n}^{\prime}\right)$, bulk concentrations of $\mathbf{C a}^{2+}$ $\left(C_{i o n}\right)$, relative surface excess of calcium with respect to water $\left(\Gamma_{C a}^{w a t e r}\right)$, and the percetages of $\mathrm{Ca}^{2+}$ bound to phosphate or carbonyl oxyges $\left(r_{\mathrm{PO}_{4}}^{\mathrm{Ca}^{2+}}\right.$ and $\left.r_{\mathrm{O}_{\text {carb. }}}^{\mathrm{Ca}^{2+}}\right)$ in different POPC bilayer models.

\begin{tabular}{l|cc|c|cc} 
model & $C_{i o n}^{\prime}$ & $C_{i o n} / \mathrm{mM}$ & $\Gamma_{C a}^{w a t e r} / \mathrm{nm}^{-2}$ & $r_{\mathrm{PO}_{4}}^{\mathrm{Ca}^{2+}}$ & $r_{\mathrm{O}_{\text {carb. }}}^{\mathrm{Ca}^{2+}}$ \\
\hline ECC-POPC & 350 & $280 \pm 10$ & $0.06 \pm 0.01$ & $99 \%$ & $32 \%$ \\
Lipid14/Åqvist & 350 & $210 \pm 10$ & $0.13 \pm 0.01$ & $100 \%$ & $37 \%$ \\
Lipid14/Dang & 350 & $160 \pm 10$ & $0.23 \pm 0.03$ & $100 \%$ & $14 \%$ \\
Lipid14/ECC-ions & 350 & $120 \pm 100$ & $0.35 \pm 0.11$ & $100 \%$ & $23 \%$
\end{tabular}

P-N vector angle are too pronounced for the Lipid14 model, for which the largest tilting toward water phase induced by a $780 \mathrm{mM} \mathrm{CaCl}_{2}$ concentration is approximately $17^{\circ}$. The corresponding value for the ECC-POPC simulation is only $6^{\circ}\left(820 \mathrm{mM} \mathrm{CaCl}_{2}\right)$.

Within the Lipid14 model, the overestimated changes in the lipid headgroup order parameter of POPC as functions of the $\mathrm{CaCl}_{2}$ concentration arise both from the overestimated binding affinity and the excessive sensitivity of the headgroup tilt to the bound positive charge. It is plausible to assume that the same applies to the other lipid models tested in a previous study, ${ }^{29}$ which underlines the importance of validation of the lipid headgroup order parameter response to the bound charge.

Finally, the ion binding affinities for the ECC-POPC model with different water models are compared in SI. In general, the performance of ECC-POPC with any of the tested water models is better than that of the original Lipid14 model, with the order parameter changes being slightly overestimated with the four-site water models and with TIP3P model.

\section{Binding affinities of $\mathrm{Na}$ and $\mathrm{Ca}$ cations to the POPC membrane}

Binding affinities of $\mathrm{Ca}^{2+}$ ions to a POPC bilayer in different simulation models were quantified by calculating the relative surface excess of calcium with respect to water molecules, $\Gamma_{\text {ion }}^{\text {water }}$, from Eq. 4. The values of $\Gamma_{\text {ion }}^{\text {water }}$ from different simulations with the same molar concetration of cations with respect to water $\left(C_{i o n}^{\prime}=350 \mathrm{mM}\right)$ are shown in Table 2. As expected from the changes of the lipid headgroup order parameters in Fig. 4, the relative surface excess of calcium, $\Gamma_{\mathrm{Ca}}^{\mathrm{water}}$ $=0.06 \mathrm{~nm}^{-2}$, is significantly smaller for the ECC-POPC model than for the other models, $0.13-$ 
$0.35 \mathrm{~nm}^{-2}$. Interestingly, the calculated relative surface excess of $\mathrm{NaCl}$ at $1 \mathrm{M}$ concentration (ECCions ${ }^{33}$ ) using our ECC-POPC model is not only quantitatively but also qualitatively different from $\mathrm{CaCl}_{2}$ having actually a negative value of $\left.\Gamma_{N a}^{\text {water }}=-0.11 \pm 0.01\right) \mathrm{nm}^{-2}$ (Fig. 5). This means that on average water molecules are preferred to sodium and chloride ions at the membrane-water interface. This is in contradiction with most of the available lipid force fields, which predict a positive surface excess of sodium at PC lipid bilayers. ${ }^{29}$

\section{Molecular interactions between $\mathrm{Na}$ or $\mathrm{Ca}$ cations and POPC oxygens}

We analyzed the ratio of the number of calcium cations bound to either phosphate or carbonyl moieties and the total number of bound cations in our POPC bilayers as done previously in Ref. 25. A maximum distance of $0.3 \mathrm{~nm}$ from any lipid oxygen is used to define a bound calcium. The results from ECC-POPC simulation in Table 2 show that almost all (99\%) of the bound $\mathrm{Ca}^{2+}$ ions are in direct contact with phosphate oxygens. From these ions, only one third (32\%) also interacts with the carbonyl oxygens, while the interaction of calcium ions with carbonyl oxygens only is rare (1\%). The most abundand interaction scenarios between $\mathrm{Ca}^{2+}$ ions and phosphate oxygens are visualized using the probability density isocontours in Fig. 6. While higher concentrations of $\mathrm{CaCl}_{2}$ increase the number of contacts per lipid, the distribution of contacts between phosphate and carbonyl oxygens is not affected.

Even though $\mathrm{Na}^{+}$ions do not bind strongly to a POPC bilayer, they still interact mostly with its oxygen moieties. The results from a simulation at a $1 \mathrm{M} \mathrm{NaCl}$ concentration show that $55 \%$ of $\mathrm{Na}^{+}$ ions at the bilayer interact with phosphate oxygens of POPC only and 20\% with carbonyl oxygens only, with the remaining $25 \%$, is interacting with both negatively charged groups.

In conclusion, the results suggest that calcium ions bind specifically to the phosphate oxygens, occasionally interacting also with the carbonyls of the PC lipids. This is in a qualitative agreement with previous conclusions from several experimental studies. ${ }^{2,12,26-28}$ However, the present results suggest, in agreement with experiments, an overally weaker binding to the bilayer, in particular with a lower relative binding affinity to the carbonyls than inferred from previous MD simulation 


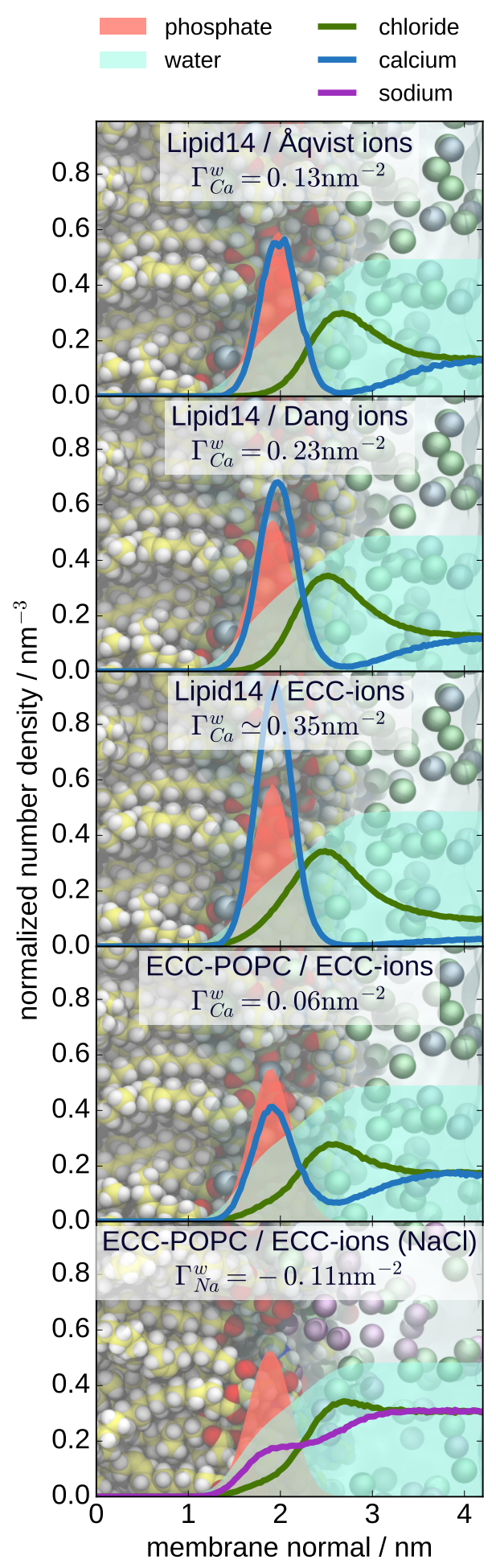

Figure 5: Number density profiles of $\mathrm{Ca}^{2+}, \mathrm{Na}^{+}$and $\mathrm{Cl}^{-}$along membrane normal axis for different force fields. In order to visualize the density profiles with a scale comparable to the profile of $\mathrm{Ca}^{2+}$, the density profiles of $\mathrm{Cl}^{-}$and $\mathrm{Na}^{+}$ions are divided by 2 , and the density profiles of phosphate groups and water are divided by 5 and 200, respectively. All simulations with $\mathrm{CaCl}_{2}$ shown here have the same molar concentration of ions in water $\left(C_{i o n}^{\prime}=350 \mathrm{mM}\right)$. The simulation with $\mathrm{NaCl}$ has $C_{\text {ion }}^{\prime}=1000 \mathrm{mM}$. 


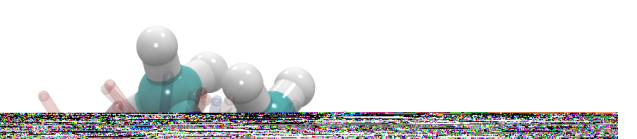

Figure 6: Isocontours of spatial number density of $\mathrm{Ca}^{2+}$ (dark blue, $0.001 \AA^{-3}$ ) and POPC carbonyl oxygen atoms (light semi-transparent red, $0.008 \AA^{-3}$, all POPC lipids contribute). Calcium cations localize mostly around phosphate oxygens (oxygens red, phosphorus bronze). Interactions with carbonyl oxygens is less likely than with phosphate oxygens, and it is contributed more by other neighbouring phospholipids than by the same lipid. Transparent structures are shown to depict the variability of choline configurations (colour warps from red to blue along the simulation time). The number density was evaluated for each lipid, after its structural alignment using only phosphate group. MDAnalysis ${ }^{101}$ library was used for the calculations of the structural alignment and the spatial number density. VMD ${ }^{102}$ was used for visualisation. Carbon atoms are depicted in cyan, hydrogen atoms in white, oxygen atoms in red, nitrogen in blue.

studies. ${ }^{14,15,24,25}$ Sodium ions, which do not exhibit any appreciable affinity for the bilayer, also interact primarily with phosphate oxygens of the POPC, but in contrast to calcium, the interactions purely with carbonyls are also significant.

\section{Binding stoichiometry of $\mathrm{Na}^{+}$and $\mathrm{Ca}^{2+}$ cations to POPC membrane}

Simple binding models have been used previously to interpret the same experimental data ${ }^{11,103}$ as employed in this work to validate the simulation models (Fig. 4). In particular, NMR data concerning the $\mathrm{PC}$ headgroup order parameters response and atomic absorption spectra were explained best using a ternary complex binding model with a binding stoichiometry of one $\mathrm{Ca}^{2+}$ per two POPC lipids. ${ }^{11}$ Nevertheless, a Langmuir adsorption model assuming a $\mathrm{Ca}^{2+}: \mathrm{POPC}$ stoichiometry of 1:1 


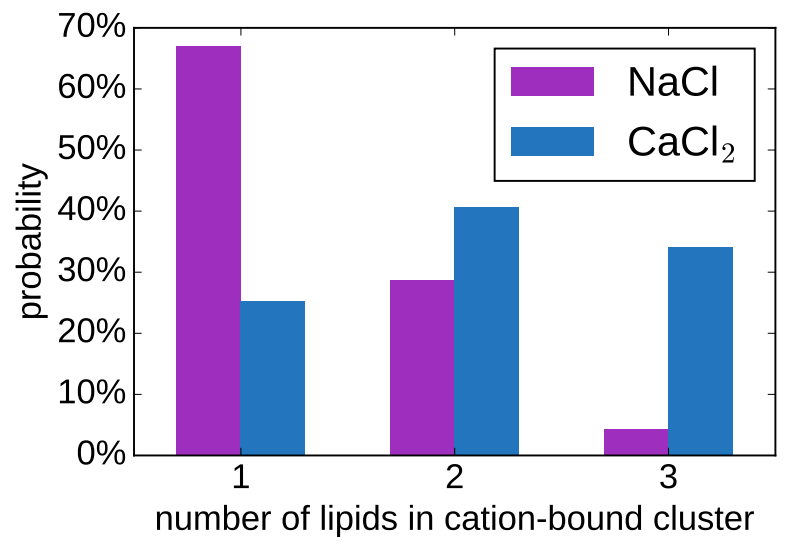

Figure 7: Relative probabilities of existence of $\mathrm{Na}^{+}$or $\mathrm{Ca}^{2+}$ complexes with a certain number of POPC lipids. $\mathrm{Na}^{+}$complexes were evaluated from the simulation with $1 \mathrm{M}$ concentration; and $\mathrm{Ca}^{2+}$ complexes were evaluated from the simulation with $287 \mathrm{mM}$ concentration.

also provided a good fit to the experimental data when considering $\mathrm{CaCl}_{2}$ at low concentrations only. ${ }^{103}$

In this work, we reproduce the same experimental data used to infer binding stoichiometries employing our ECC-POPC model. Thanks to our simulations, we have a direct access to atomistic details of the binding stoichiometry without a need for any binding model as employed for interpreting in experiments. ${ }^{11,103}$ To evaluate the relative propensities for each of the stoichiometric complexes (i.e., $1 \mathrm{Ca}^{2+}:$ n POPC), we calculated for each bound $\mathrm{Ca}^{2+}$ the number of POPC molecules having oxygen atoms within a distance of $0.3 \mathrm{~nm}$. Results from the POPC bilayer simulation with a $285 \mathrm{mM}$ bulk concentration of $\mathrm{CaCl}_{2}$ are shown in Fig. 7. We found the largest propensity for the 1:2 complex (41\%), with probabilities of complexes with the stoichiometries of 1:1 (25\%) and 1:3 (34\%) being only slightly lower. This suggests a more complex binding model than considered in a simple 1:2 ternary complex model previously. Nevertheless, with a broad brushstroke, the simulation data can be viewed such that one calcium binds to two lipids on average, because the probabilities of the complexes with 1 or 3 lipids are almost equal to each other (and complexes with more than three lipids per one calcium ion were not observed). This probably explains why the simple the ternary complex model fits adequately the experimental data, as well as the ECC-POPC simulation results (see Fig. S3 in SI). 
The probabilities of different complexes formed by $\mathrm{Na}^{+}$ions and POPC analyzed from the simulation with the ECC-POPC model at $1 \mathrm{M}$ concentration of $\mathrm{NaCl}$ are also shown in Fig. 7. In contrast to calcium, the probability is largest (67\%) for 1:1 complex, significantly smaller (29\%) for $1: 2$ complexes and very small $(4 \%)$ for $1: 3$ of $\mathrm{Na}^{+}$:POPC complexes.

\section{Residence times of $\mathrm{Na}^{+}$and $\mathrm{Ca}^{2+}$ cations in the POPC membrane}

Equilibration of $\mathrm{Ca}^{2+}$ ions at a POPC bilayer in MD simulations is a microsecond time scale process with current force fields, such as CHARMM36 and Slipids force fields. ${ }^{25}$ This suggests that at least several microseconds are required to reach the ion binding/unbinding equilibrium. To quantify the exchange of ions between the membrane and aqueous solution in simulations, we evaluated residence times of ions bound to the membrane. Within our analysis, an ion is considered to be bound when it is within $0.3 \mathrm{~nm}$ from any oxygen atom belonging to a POPC molecule.

The histograms of residence times of $\mathrm{Ca}^{2+}$ in a POPC bilayer $\left(C_{i o n}^{\prime}=450 \mathrm{mM}\right)$ from simulations with ECC-POPC and CHARMM36 (simulation from Refs. 25,104) are shown in Fig. S4 in SI. In the CHARMM36 simulation, a significant number of the calcium ions is bound to the membrane for the whole length of the trajectory $(800 \mathrm{~ns})$. In contrast, at least an order of magnitude faster bound/unbound calcium exchange is observed within the ECC-POPC model, where $90 \%$ of the $\mathrm{Ca}^{2+}$ residence times to a POPC membrane are shorter than $60 \mathrm{~ns}$. The longest observed residence time is around $150 \mathrm{~ns}$, which is below the total length of the simulation used for analysis, i.e., 200 ns. Note that these results are in line with the experimental estimate that the residence time of $\mathrm{Ca}^{2+}$ at each PC headgroup is less than $10 \mu \mathrm{s} .{ }^{11}$ Note that the exchange of $\mathrm{Na}^{+}$ions at the POPC membrane is yet another order of magnitude faster, with $90 \%$ of the residence times smaller than $1 \mathrm{~ns}$ and the longest residence time being $6 \mathrm{~ns}$.

In summary, the results from the ECC-POPC model suggest that the exchange of calcium between the POPC bilayer and the solvent occurs within the $\sim 100$ ns timeframe, which is significantly faster than observed in simulations emloying most of the presently available lipid models. ${ }^{25}$ Sodium cations exhibit an even more rapid exchange between the membrane and the aqueous so- 
lution. Our results suggest that simulations with a length of several hundreds of nanoseconds are sufficient to simulate alkali and alkali earth ion binding to phospholipid bilayers in equilibrium when realistic force fields are used. This has not been the case with previous lipid force fields, which overestimate the binding strength of the sodium and, in particular, calcium cations. ${ }^{25,29}$

\section{Conclusions}

In this study, we employed the electrometer concept to demonstrate that the binding of $\mathrm{Na}^{+}$and $\mathrm{Ca}^{2+}$ ions to a POPC lipid bilayer can be accurately described within a force field MD simulation, provided that electronic polarization is implicitly included via the electronic continuum correction. ${ }^{31}$ This ECC-POPC model is built upon the Lipid14 POPC model ${ }^{36}$ by scaling the partial charges by a factor of 0.8 and reducing the Lennard-Jones radii by a factor of 0.89 for the headgroup, glycerol backbone, and carbonyl atoms. While the structural details of a POPC lipid bilayer in pure water simulated with the newly developed ECC-POPC model agree with experiments with an accuracy comparable to the other state of the art lipid models, the new model in addition reproduces the experimental lipid head group order parameter responses to a cationic surfactant, $\mathrm{NaCl}$, and $\mathrm{CaCl}_{2}$ at varying concentrations. It thus represents a significant improvement over currently available lipid models, which tend to overestimate cation binding affinities. ${ }^{29}$ A similar improvement using the ECC procedure is demonstrated for the POPC model of CHARMM36 ${ }^{53}$ in the SI.

The good agreement with experiments enables us to interpret NMR experiments with atomistic details using MD simulations with the ECC-POPC model. In line with previous interpretations of the experimental data, ${ }^{12,26-28} \mathrm{Ca}^{2+}$ ions interact mainly with phosphate oxygens, with interactions to the carbonyls being of a secondary importance. However, the stoichiometry of calcium binding is significantly more complicated than the simple ternary complex model, used to interpret NMR data previously, within which one calcium binds to two POPC molecules. ${ }^{11}$ While complexes with one calcium ion bound to two lipids are the most probable also in the ECC-POPC model, 
complexes of one or three lipids per one calcium were observed to be relatively abundant and also almost equally likely to occur. While the success of the simple ternary complex model in fitting NMR data is understandable based on the simulation results, it cannot capture the detailed nature of calcium binding to phospholipid bilayers observed in the present simulation.

Accurate description of cation binding to POPC bilayer paves the way for simulations of complex biochemical systems at cellular membranes with realistically described electrostatic interactions, including a mean-field account for electronic polarization effects. To this end, the compatibility of the ECC-POPC model with existing models for proteins and other biological molecules needs to be verified and, if necessary, further adjustments following the ECC concept to the force fields of the biomolecules interacting with the lipids should be made. In future studies, the ECC concept will be applied also to bilayers composed of other lipids than POPC.

This work can be reached as a repository containing all data and scripts for analysis at Ref. 105.

\section{Acknowledgement}

P.J. acknowledges support from the Czech Science Foundation (grant no. 16-01074S) and from the Academy of Finland via the FiDiPro award. J.K. acknowledges support from the Czech Science Foundation, Project No. 15-12386S. Computational resources were supplied by the Ministry of Education, Youth and Sports of the Czech Republic under the Projects CESNET (Project No. LM2015042) and CERIT-Scientific Cloud (Project No. LM2015085) provided within the program Projects of Large Research, Development and Innovations Infrastructures. O.H.S.O. acknowledges financial support from Integrated Structural Biology Research Infrastructure of Helsinki Institute of Life Science (Instruct-HiLIFE).

\section{Supporting Information Available}

The following files are available free of charge. 
- SI.pdf: Simulation details and further analysis.

This material is available free of charge via the Internet at http://pubs.acs.org/.

\section{References}

(1) Seelig, J. Interaction of phospholipids with $\mathrm{Ca} 2+$ ions. On the role of the phospholipid head groups. Cell Biol. Int. Rep. 1990, 14, 353-360.

(2) Cevc, G. Membrane electrostatics. Biochim. Biophys. Acta 1990, 1031, 311 - 382.

(3) Tocanne, J.-F.; Teissié, J. Ionization of phospholipids and phospholipid-supported interfacial lateral diffusion of protons in membrane model systems. Biochim. Biophys. Acta 1990, $1031,111-142$.

(4) Pabst, G.; Hodzic, A.; Strancar, J.; Danner, S.; Rappolt, M.; Laggner, P. Rigidification of neutral lipid bilayers in the presence of salts. Biophys. J. 2007, 93, $2688-2696$.

(5) Papahadjopoulos, D.; Nir, S.; Düzgünes, N. Molecular mechanisms of calcium-induced membrane fusion. J. Bioenerg. Biomembr. 1990, 22, 157-179.

(6) Brouwer, I.; Giniatullina, A.; Laurens, N.; van Weering, J. R. T.; Bald, D.; Wuite, G. J. L.; Groffen, A. J. Direct quantitative detection of Doc2b-induced hemifusion in optically trapped membranes. Nat. Comm. 2015, 6, 8387.

(7) Shi, X.; Bi, Y.; Yang, W.; Guo, X.; Jiang, Y.; Wan, C.; Li, L.; Bai, Y.; Guo, J.; Wang, Y. et al. $\mathrm{Ca} 2+$ regulates $\mathrm{T}$-cell receptor activation by modulating the charge property of lipids. Nature 2013, 493, 111-115.

(8) Bilkova, E.; Pleskot, R.; Rissanen, S.; Sun, S.; Czogalla, A.; Cwiklik, L.; Róg, T.; Vattulainen, I.; Cremer, P. S.; Jungwirth, P. et al. Calcium directly regulates phosphatidylinositol 4,5-bisphosphate headgroup conformation and recognition. J. Am. Chem. Soc. 2017, 139, 4019-4024. 
(9) Scherer, P.; Seelig, J. Structure and dynamics of the phosphatidylcholine and the phosphatidylethanolamine head group in L-M fibroblasts as studied by deuterium nuclear magnetic resonance. EMBO J. 1987, 6, 2915-2922.

(10) Akutsu, H.; Seelig, J. Interaction of metal ions with phosphatidylcholine bilayer membranes. Biochemistry 1981, 20, 7366-7373.

(11) Altenbach, C.; Seelig, J. Calcium binding to phosphatidylcholine bilayers as studied by deuterium magnetic resonance. Evidence for the formation of a calcium complex with two phospholipid molecules. Biochemistry 1984, 23, 3913-3920.

(12) Binder, H.; Zschörnig, O. The effect of metal cations on the phase behavior and hydration characteristics of phospholipid membranes. Chem. Phys. Lipids 2002, 115, 39 - 61.

(13) Uhríková, D.; Kučerka, N.; Teixeira, J.; Gordeliy, V.; Balgavý, P. Structural changes in dipalmitoylphosphatidylcholine bilayer promoted by $\mathrm{Ca} 2+$ ions: a small-angle neutron scattering study. Chem. Phys. Lipids 2008, 155, $80-89$.

(14) Böckmann, R. A.; Hac, A.; Heimburg, T.; Grubmüller, H. Effect of sodium chloride on a lipid bilayer. Biophys. J. 2003, 85, $1647-1655$.

(15) Böckmann, R. A.; Grubmüller, H. Multistep binding of divalent cations to phospholipid bilayers: a molecular dynamics study. Ang. Chem. Int. Ed. 2004, 43, 1021-1024.

(16) Sachs, J. N.; Nanda, H.; Petrache, H. I.; Woolf, T. B. Changes in phosphatidylcholine headgroup tilt and water order induced by monovalent salts: Molecular dynamics simulations. Biophys. J. 2004, 86, $3772-3782$.

(17) Berkowitz, M. L.; Bostick, D. L.; Pandit, S. Aqueous solutions next to phospholipid membrane surfaces: Insights from simulations. Chem. Rev. 2006, 106, 1527-1539.

(18) Cordomí, A.; Edholm, O.; Perez, J. J. Effect of ions on a dipalmitoyl phosphatidylcholine bilayer. A molecular dynamics simulation study. J. Phys. Chem. B 2008, 112, 1397-1408. 
(19) Cordomí, A.; Edholm, O.; Perez, J. J. Effect of force field parameters on sodium and potassium ion binding to dipalmitoyl phosphatidylcholine bilayers. J. Chem. Theory Comput. 2009, 5, 2125-2134.

(20) Vacha, R.; Siu, S. W. I.; Petrov, M.; Böckmann, R. A.; Barucha-Kraszewska, J.; Jurkiewicz, P.; Hof, M.; Berkowitz, M. L.; Jungwirth, P. Effects of alkali cations and halide anions on the DOPC lipid membrane. J. Phys. Chem. A 2009, 113, 7235-7243.

(21) Valley, C.; Perlmutter, J.; Braun, A.; Sachs, J. NaCl interactions with phosphatidylcholine bilayers do not alter membrane structure but induce long-range ordering of ions and water. J. Membr. Biol. 2011, 244, 35-42.

(22) Berkowitz, M. L.; Vacha, R. Aqueous solutions at the interface with phospholipid bilayers. Acc. Chem. Res. 2012, 45, 74-82.

(23) Knecht, V.; Klasczyk, B. Specific binding of chloride ions to lipid vesicles and implications at molecular scale. Biophys. J. 2013, 104, 818 - 824.

(24) Melcrová, A.; Pokorna, S.; Pullanchery, S.; Kohagen, M.; Jurkiewicz, P.; Hof, M.; Jungwirth, P.; Cremer, P. S.; Cwiklik, L. The complex nature of calcium cation interactions with phospholipid bilayers. Sci. Reports 2016, 6, 38035.

(25) Javanainen, M.; Melcrová, A.; Magarkar, A.; Jurkiewicz, P.; Hof, M.; Jungwirth, P.; Martinez-Seara, H. Two cations, two mechanisms: interactions of sodium and calcium with zwitterionic lipid membranes. Chem. Commun. 2017, 53, 5380-5383.

(26) Hauser, H.; Phillips, M. C.; Levine, B.; Williams, R. Conformation of the lecithin polar group in charged vesicles. Nature 1976, 261, 390 - 394.

(27) Hauser, H.; Guyer, W.; Levine, B.; Skrabal, P.; Williams, R. The conformation of the polar group of lysophosphatidylcholine in $\mathrm{H} 2 \mathrm{O}$; conformational changes induced by polyvalent cations. Biochim. Biophys. Acta 1978, 508, 450 - 463. 
(28) Herbette, L.; Napolitano, C.; McDaniel, R. Direct determination of the calcium profile structure for dipalmitoyllecithin multilayers using neutron diffraction. Biophys. J. 1984, 46, 677 $-685$.

(29) Catte, A.; Girych, M.; Javanainen, M.; Loison, C.; Melcr, J.; Miettinen, M. S.; Monticelli, L.; Maatta, J.; Oganesyan, V. S.; Ollila, O. H. S. et al. Molecular electrometer and binding of cations to phospholipid bilayers. Phys. Chem. Chem. Phys. 2016, 18, 3256032569.

(30) Seelig, J.; MacDonald, P. M.; Scherer, P. G. Phospholipid head groups as sensors of electric charge in membranes. Biochemistry 1987, 26, 7535-7541.

(31) Leontyev, I.; Stuchebrukhov, A. Accounting for electronic polarization in non-polarizable force fields. Phys. Chem. Chem. Phys. 2011, 13, 2613-2626.

(32) Martinek, T.; Duboué-Dijon, E.; Timr, S.; Mason, P. E.; Baxová, K.; Fischer, H. E.; Schmidt, B.; Pluhařová, E.; Jungwirth, P. Calcium ions in aqueous solutions: Accurate force field description aided by ab initio molecular dynamics and neutron scattering. J. Chem. Phys. 2018, 148, 222813.

(33) Pluhařová, E.; Fischer, H. E.; Mason, P. E.; Jungwirth, P. Hydration of the chloride ion in concentrated aqueous solutions using neutron scattering and molecular dynamics. Mol. Phys. 2014, 112, 1230-1240.

(34) Kohagen, M.; Mason, P. E.; Jungwirth, P. Accurate description of calcium solvation in concentrated aqueous solutions. J. Phys. Chem. B 2014, 118, 7902-7909.

(35) Kohagen, M.; Mason, P. E.; Jungwirth, P. Accounting for electronic polarization effects in aqueous sodium chloride via molecular dynamics aided by neutron scattering. J. Phys. Chem. B 2016, 120, 1454-1460. 
(36) Dickson, C. J.; Madej, B. D.; Skjevik, . A.; Betz, R. M.; Teigen, K.; Gould, I. R.; Walker, R. C. Lipid14: The amber lipid force field. J. Chem. Theory Comput. 2014, 10, $865-879$.

(37) Lucas, T. R.; Bauer, B. A.; Patel, S. Charge equilibration force fields for molecular dynamics simulations of lipids, bilayers, and integral membrane protein systems. Biochim. Biophys. Acta 2012, 1818, $318-329$.

(38) Chowdhary, J.; Harder, E.; Lopes, P. E. M.; Huang, L.; MacKerell, A. D.; Roux, B. A polarizable force field of dipalmitoylphosphatidylcholine based on the classical drude model for molecular dynamics simulations of lipids. J. Phys. Chem. B 2013, 117, 9142-9160.

(39) Jönsson, B.; Edholm, O.; Teleman, O. Molecular dynamics simulations of a sodium octanoate micelle in aqueous solution. J. Chem. Phys. 1986, 85, 2259-2271.

(40) Egberts, E.; Marrink, S.-J.; Berendsen, H. J. C. Molecular dynamics simulation of a phospholipid membrane. Eur. Biophys. J. 1994, 22, 423-436.

(41) Beichel, W.; Trapp, N.; Hauf, C.; Kohler, O.; Eickerling, G.; Scherer, W.; Krossing, I. Charge-scaling effect in ionic liquids from the charge-density analysis of N,Ndimethylimidazolium methylsulfate. Ang. Chem. Int. Ed. 2014, 53, 3143-3146.

(42) Leontyev, I. V.; Stuchebrukhov, A. A. Electronic continuum model for molecular dynamics simulations. J. Chem. Phys. 2009, 130, 085102.

(43) Leontyev, I. V.; Stuchebrukhov, A. A. Electronic continuum model for molecular dynamics simulations of biological molecules. J. Chem. Theory Comput. 2010, 6, 1498-1508.

(44) Leontyev, I. V.; Stuchebrukhov, A. A. Polarizable molecular interactions in condensed phase and their equivalent nonpolarizable models. J. Chem. Phys. 2014, 141, 014103.

(45) Hu, H.; Lu, Z.; and Weitao Yang, Fitting molecular electrostatic potentials from quantum mechanical calculations. J. Chem. Theory Comput. 2007, 3, 1004-1013. 
(46) Bayly, C. C. I.; Cieplak, P.; Cornell, W. D.; Kollman, P. A. A well-behaved electrostatic potential based method using charge restraints for deriving atomic charges: the RESP model. J. Phys. Chem. 1993, 97, 10269-10280.

(47) Singh, U. C.; Kollman, P. A. An approach to computing electrostatic charges for molecules. J. Comput. Chem. 1984, 5, 129-145.

(48) Jorgensen, W. L.; Maxwell, D. S.; Tirado-Rives, J. Development and testing of the OPLS all-atom force field on conformational energetics and properties of organic liquids. J. Am. Chem. Soc. 1996, 118, 11225-11236.

(49) Cerutti, D. S.; Rice, J. E.; Swope, W. C.; Case, D. A. Derivation of fixed partial charges for amino acids accommodating a specific water model and implicit polarization. J. Phys. Chem. B 2013, 117, 2328-2338.

(50) Benavides, A. L.; Portillo, M. A.; Chamorro, V. C.; Espinosa, J. R.; Abascal, J. L. F.; Vega, C. A potential model for sodium chloride solutions based on the TIP4P/2005 water model. J. Chem. Phys. 2017, 147, 104501.

(51) Ollila, O. H. S.; Retegan, M. MD simulation trajectory and related files for POPC bilayer (Lipid14, Gromacs 4.5). 2014; http://dx.doi.org/10.5281/zenodo.12767.

(52) Botan, A.; Favela-Rosales, F.; Fuchs, P. F. J.; Javanainen, M.; Kanduč, M.; Kulig, W.; Lamberg, A.; Loison, C.; Lyubartsev, A.; Miettinen, M. S. et al. Toward atomistic resolution structure of phosphatidylcholine headgroup and glycerol backbone at different ambient conditions. J. Phys. Chem. B 2015, 119, 15075-15088.

(53) Klauda, J. B.; Venable, R. M.; Freites, J. A.; O’Connor, J. W.; Tobias, D. J.; MondragonRamirez, C.; Vorobyov, I.; Jr, A. D. M.; Pastor, R. W. Update of the CHARMM all-atom additive force field for lipids: Validation on six lipid types. J. Phys. Chem. B 2010, 114, $7830-7843$. 
(54) Kann, Z. R.; Skinner, J. L. A scaled-ionic-charge simulation model that reproduces enhanced and suppressed water diffusion in aqueous salt solutions. J. Chem. Phys. 2014, 141, 104507.

(55) Fuentes-Azcatl, R.; Barbosa, M. C. Sodium chloride, $\mathrm{NaCl} / \epsilon$ : New force field. J. Phys. Chem. B 2016, 120, 2460-2470.

(56) Dommert, F.; Wendler, K.; Qiao, B.; Delle Site, L.; Holm, C. Generic force fields for ionic liquids. J. Mol. Liq. 2014, 192, 32-37.

(57) Kučerka, N.; Nieh, M. P.; Katsaras, J. Fluid phase lipid areas and bilayer thicknesses of commonly used phosphatidylcholines as a function of temperature. Biochim. Biophys. Acta 2011, 1808, 2761-2771.

(58) Ollila, O. S.; Pabst, G. Atomistic resolution structure and dynamics of lipid bilayers in simulations and experiments. Biochim. Biophys. Acta 2016, 1858, 2512 - 2528.

(59) Ferreira, T. M.; Sood, R.; Bärenwald, R.; Carlström, G.; Topgaard, D.; Saalwächter, K.; Kinnunen, P. K. J.; Ollila, O. H. S. Acyl chain disorder and azelaoyl orientation in lipid membranes containing oxidized lipids. Langmuir 2016, 32, 6524-6533.

(60) Beschiaschvili, G.; Seelig, J. Peptide binding to lipid membranes. Spectroscopic studies on the insertion of a cyclic somatostatin analog into phospholipid bilayers. Biochim. Biophys. Acta 1991, 1061, $78-84$.

(61) Scherer, P. G.; Seelig, J. Electric charge effects on phospholipid headgroups. Phosphatidylcholine in mixtures with cationic and anionic amphiphiles. Biochemistry 1989, 28, 77207728.

(62) Chattoraj, D. K.; Birdi, K. S. Adsorption and the Gibbs Surface Excess; Springer US: Boston, MA, 1984; pp 83-131. 
(63) Berendsen, H. J. C.; Grigera, J. R.; Straatsma, T. P. The missing term in effective pair potentials. J. Phys. Chem. 1987, 91, 6269-6271.

(64) Izadi, S.; Anandakrishnan, R.; Onufriev, A. V. Building water models: A different approach. J. Phys. Chem. Lett. 2014, 5, 3863-3871.

(65) Izadi, S.; Onufriev, A. V. Accuracy limit of rigid 3-point water models. J. Chem. Phys. 2016, $145,074501$.

(66) Jorgensen, W. L.; Chandrasekhar, J.; Madura, J. D.; Impey, R. W.; Klein, M. L. Comparison of simple potential functions for simulating liquid water. J. Chem. Phys 1983, 79, 926-935.

(67) Wang, L. P.; Martinez, T. J.; Pande, V. S. Building force fields: An automatic, systematic, and reproducible approach. Phys. Chem. Lett. 2014, 5, 1885-1891.

(68) Abascal, J. L.; Vega, C. A general purpose model for the condensed phases of water: TIP4P/2005. J. Chem. Phys. 2005, 123, 234505.

(69) Smith, D. E.; Dang, L. X. Computer simulations of $\mathrm{NaCl}$ association in polarizable water. J. Chem. Phys 1994, 100, 3757-3766.

(70) Chang, T.-M.; Dang, L. X. Detailed study of potassium solvation using molecular dynamics techniques. J. Phys. Chem. B 1999, 103, 4714-4720.

(71) Dang, L. X.; Schenter, G. K.; Glezakou, V.-A.; Fulton, J. L. Molecular simulation analysis and X-ray absorption measurement of $\mathrm{Ca} 2+, \mathrm{K}+$ and $\mathrm{Cl}-$ ions in solution. J. Phys. Chem. B 2006, 110, 23644-54.

(72) Aaqvist, J. Ion-water interaction potentials derived from free energy perturbation simulations. J. Phys. Chem. 1990, 94, 8021-8024.

(73) Girych, M.; Ollila, O. H. S. POPC_AMBER_LIPID14_Verlet. 2015; http: / / dx • doi . org/10.5281/zenodo.30898. 
(74) Girych, M.; Ollila, O. H. S. POPC_AMBER_LIPID14_CaCl2_035Mol. 2015; http:// dx.doi.org/10.5281/zenodo.34415.

(75) Girych, M.; Ollila, S. POPC_AMBER_LIPID14_CaCl2_035Mol. 2016; https ://doi . org/10.5281/zenodo.46234.

(76) Girych, M.; Ollila, O. H. S. POPC_AMBER_LIPID14_NaCl_1Mol. 2015; http://dx . doi.org/10.5281/zenodo.30865.

(77) Abraham, M. J.; Murtola, T.; Schulz, R.; Páll, S.; Smith, J. C.; Hess, B.; Lindah, E. Gromacs: High performance molecular simulations through multi-level parallelism from laptops to supercomputers. SoftwareX 2015, 1-2, 19-25.

(78) Eastman, P.; Swails, J.; Chodera, J. D.; McGibbon, R. T.; Zhao, Y.; Beauchamp, K. A.; Wang, L.-P.; Simmonett, A. C.; Harrigan, M. P.; Stern, C. D. et al. OpenMM 7: Rapid development of high performance algorithms for molecular dynamics. PLOS Comput. Biol. 2017, 13, e1005659.

(79) Melcr, J. Simulations of a POPC lipid bilayer in water solution at various $\mathrm{NaCl}$ and $\mathrm{CaCl} 2$ concentration with Lipid14, TIP3p and Dang or ECC ions. 2017; https://doi.org/ 10.5281 /zenodo.1108568.

(80) Melcr, J. Simulations of POPC lipid bilayer in water solution at various $\mathrm{NaCl}$ and $\mathrm{CaCl} 2$ concentrations using ECC-POPC force field. 2017; https://doi.org/10.5281/ zenodo.1118265.

(81) Melcr, J. Simulations of POPC lipid bilayer in water solution at various $\mathrm{NaCl}$ and $\mathrm{CaCl} 2$ concentrations using ECC-POPC force field and various water models . 2017; https: //doi.org/10.5281/zenodo.1118979.

(82) Malde, A. K.; Zuo, L.; Breeze, M.; Stroet, M.; Poger, D.; Nair, P. C.; Oostenbrink, C.; 
Mark, A. E. An automated force field topology builder (ATB) and repository: Version 1.0. J. Chem. Theory Comput. 2011, 7, 4026-4037.

(83) Case, D.; Cerutti, D.; Cheatham, T.; III,; Darden, T.; Duke, R.; Giese, T.; Gohlke, H.; Goetz, A.; Greene, D. et al. AMBER 2017. 2017; University of California, San Francisco.

(84) Sousa da Silva, A. W.; Vranken, W. F. ACPYPE - AnteChamber PYthon Parser interfacE. BMC Res. Notes 2012, 5, 367.

(85) Melcr, J. Simulations of POPC lipid bilayer in water solution with various molar fractions of cationic surfactant dihexadecylammonium using ECC-POPC force field. 2017; https : //doi.org/10.5281/zenodo.1118681.

(86) Ollila, O. H. S. POPC bilayer simulated at T313K with the Lipid14 model using Gromacs. 2017; https://doi.org/10.5281/zenodo.1020709.

(87) Ollila, O. H. S. POPC bilayer with $10 \%$ of dihexadecyldimethylammonium simulated at T313K with the Lipid14 model using Gromacs. 2017; https: / / doi . org/10.5281/ zenodo.1020240.

(88) Ollila, O. H. S. POPC bilayer with $20 \%$ of dihexadecyldimethylammonium simulated at T313K with the Lipid14 model using Gromacs. 2017; https : / doi . org/10.5281/ zenodo.1020593.

(89) Ollila, O. H. S. POPC bilayer with $30 \%$ of dihexadecyldimethylammonium simulated at T313K with the Lipid14 model using Gromacs. 2017; https : / / doi . org/10.5281/ zenodo.1020623.

(90) Ollila, O. H. S. POPC bilayer with $42 \%$ of dihexadecyldimethylammonium simulated at T313K with the Lipid14 model using Gromacs. 2017; https : / / doi .org/10.5281/ zenodo.1020671. 
(91) Ollila, O. H. S. POPC bilayer with $50 \%$ of dihexadecyldimethylammonium simulated at T313K with the Lipid14 model using Gromacs. 2017; https: / / doi . org/10.5281/ zenodo.1020689.

(92) Ferreira, T. M.; Coreta-Gomes, F.; Ollila, O. H. S.; Moreno, M. J.; Vaz, W. L. C.; Topgaard, D. Cholesterol and POPC segmental order parameters in lipid membranes: solid state 1H-13C NMR and MD simulation studies. Phys. Chem. Chem. Phys. 2013, 15, 1976-1989.

(93) Jones, E.; Oliphant, T.; Peterson, P.; et. al., SciPy: Open source scientific tools for Python. 2001-; http: / /www. scipy . org/, [Online; accessed Dec-2017].

(94) Seelig, A.; Seelig, J. Bilayers of dipalmitoyl-3-sn-phosphatidylcholine: Conformational differences between the fatty acyl chains. Biochim. Biophys. Acta 1975, 406, 1 - 5 .

(95) Schindler, H.; Seelig, J. Deuterium order parameters in relation to thermodynamic properties of a phospholipid bilayer. Statistical mechanical interpretati on. Biochemistry 1975, 14, 2283-2287.

(96) Gawrisch, K.; Ruston, D.; Zimmerberg, J.; Parsegian, V.; Rand, R.; Fuller, N. Membrane dipole potentials, hydration forces, and the ordering of water at membrane surfaces. Biophys. J. 1992, 61, $1213-1223$.

(97) Gurtovenko, A. A.; Miettinen, M.; ; Karttunen, M.; Vattulainen, I. Effect of monovalent salt on cationic lipid membranes as revealed by molecular dynamics simulations. J. Phys. Chem. B 2005, 109, 21126-21134.

(98) Zhao, W.; Gurtovenko, A. A.; Vattulainen, I.; Karttunen, M. Cationic dimyristoylphosphatidylcholine and dioleoyloxytrimethylammonium propane lipid bilayers: Atomistic insight for structure and dynamics. J. Phys. Chem. B 2012, 116, 269-276.

(99) Eisenberg, M.; Gresalfi, T.; Riccio, T.; McLaughlin, S. Adsorption of monovalent cations to bilayer membranes containing negative phospholipids. Biochemistry 1979, 18, 5213-5223. 
(100) Klasczyk, B.; Knecht, V.; Lipowsky, R.; Dimova, R. Interactions of alkali metal chlorides with phosphatidylcholine vesicles. Langmuir 2010, 26, 18951-18958.

(101) Michaud-Agrawal, N.; Denning, E. J.; Woolf, T. B.; Beckstein, O. MDAnalysis: A toolkit for the analysis of molecular dynamics simulations. Journal of Computational Chemistry 2011, 32, 2319-2327.

(102) Humphrey, W.; Dalke, A.; Schulten, K. VMD - Visual Molecular Dynamics. J. Mol. Graphics 1996, 14, 33-38.

(103) Macdonald, P. M.; Seelig, J. Calcium binding to mixed phosphatidylglycerolphosphatidylcholine bilayers as studied by deuterium nuclear magnetic resonance. Biochemistry 1987, 26, 1231-1240.

(104) Javanainen, M. POPC with varying amounts of cholesterol, $450 \mathrm{mM}$ of CaCl_2. Charmm36 with ECC-scaled ions. 2017; https: / / doi .org/10.5281/zenodo.259376.

(105) Melcr, J.; Ollila, O. H. S.; Martinez-Seara, H. ohsOllila/NMRlipids_VI-NewIonModel: Second submission to J. Phys. Chem. B. 2017; https: // doi .org/10.5281/zenodo . 1203882. 


\section{Graphical TOC Entry}

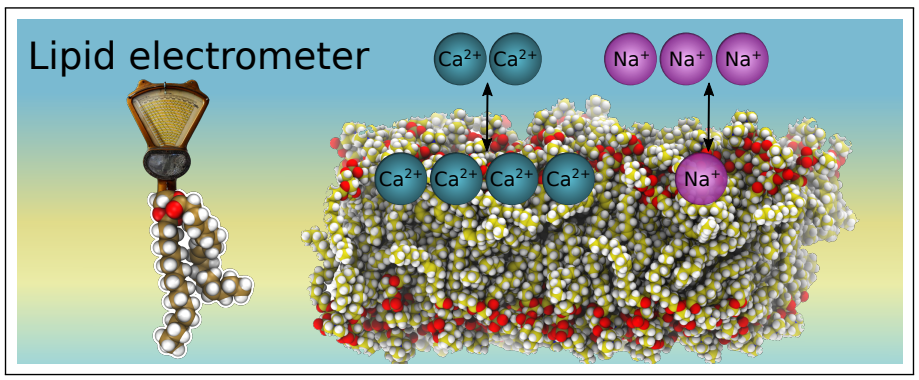

